\title{
FDTD Analysis of Electromagnetic Wave Propagation in an Inhomogeneous Ionosphere under Arbitrary-Direction Geomagnetic Field
}

\author{
Jun-Ho Kweon · Min-Seok Park · Jeahoon Cho · Kyung-Young Jung*
}

\begin{abstract}
The finite-difference time-domain (FDTD) model was developed to analyze electromagnetic (EM) wave propagation in an inhomogeneous ionosphere. The EM analysis of ionosphere is complicated, owing to various propagation environments that are significantly influenced by plasma frequency, cyclotron frequency, and collision frequency. Based on the simple auxiliary differential equation (ADE) technique, we present an accurate FDTD algorithm suitable for the EM analysis of complex phenomena in the ionosphere under arbitrarydirection geomagnetic field. Numerical examples are used to validate our FDTD model in terms of the reflection coefficient of a single magnetized plasma slab. Based on the FDTD formulation developed here, we investigate EM wave propagation characteristics in the ionosphere using realistic ionospheric data for South Korea.
\end{abstract}

Key Words: Electromagnetic Wave Propagation, Finite-Difference Time-Domain, Ionosphere.

\section{INTRODUCTION}

It is of great importance for radio and satellite communication to study electromagnetic (EM) wave propagation in the atmosphere. However, it is not easy to investigate EM wave propagation in the ionosphere because of ionized ions and free electrons [1]. Three key parameters to consider for EM analysis of the ionosphere are plasma frequency, cyclotron frequency, and collision frequency [1]. Plasma frequency is oscillation frequency, which is related to electron movement to neutralize locally biased space. Cyclotron frequency is angular frequency of electrons when they move in circular form under a static geomagnetic field. Collision frequency is the effective number of collisions per second.

In this study, we develop a finite-difference time-domain
(FDTD) [2] model suitable for EM analysis of the ionosphere, based on a simple and accurate auxiliary differential equation (ADE). Our model can accurately investigate EM wave interaction with the ionosphere because it can take into account arbitrary geomagnetic field and inhomogeneous ionospheric parameters varying in height, unlike previous FDTD studies [3]. The proposed ADE-FDTD model is validated by analyzing a simple slab of magnetized plasma. We examine EM wave interaction with the ionosphere based on realistic ionospheric data for South Korea.

\section{1-D FDTD FORMULATION}

EM interaction with magnetized plasma can be analyzed using Maxwell's equations coupled with the Lorentz equation of 
motion [1]. The governing equation set is given by the following:

$$
\begin{gathered}
\nabla \times \mathbf{H}=\varepsilon_{0} \frac{\partial \mathbf{E}}{\partial t}+\mathbf{J} \\
\nabla \times \mathbf{E}=-\mu_{0} \frac{\partial \mathbf{H}}{\partial t} \\
\frac{\partial \mathbf{J}}{\partial t}+v \mathbf{J}=\varepsilon_{0} \omega_{p}^{2} \mathbf{E}+\boldsymbol{\omega}_{b} \times \mathbf{J} .
\end{gathered}
$$

Here, $\mathrm{E}$ is the electric field, $\mathrm{H}$ is the magnetic field, $\mathbf{J}$ is the current density, $v$ is the collision frequency, $\omega_{p}$ is the plasma frequency, $\boldsymbol{\omega}_{b}$ is the cyclotron frequency, and $\varepsilon_{0}$ and $\mu_{0}$ are the permittivity and permeability of free space, respectively. For simplicity and without loss of generality, we assume that the EM wave propagates along the $z$-axis. By using the central difference scheme in both time and space [4], one can obtain

$$
\begin{gathered}
\left.H_{x}\right|_{k+1 / 2} ^{n+1 / 2}=\left.H_{x}\right|_{k+1 / 2} ^{n-1 / 2}+\frac{\Delta t}{\mu_{0} \Delta z}\left(\left.E_{y}\right|_{k+1} ^{n}-\left.E_{y}\right|_{k} ^{n}\right) \\
\left.H_{y}\right|_{k+1 / 2} ^{n+1 / 2}=\left.H_{y}\right|_{k+1 / 2} ^{n-1 / 2}-\frac{\Delta t}{\mu_{0} \Delta z}\left(\left.E_{x}\right|_{k+1} ^{n}-\left.E_{x}\right|_{k} ^{n}\right) \\
\left.E_{x}\right|_{k} ^{n+1}=\left.E_{x}\right|_{k} ^{n}-\frac{\Delta t}{\varepsilon_{0} \Delta z}\left(\left.H_{y}\right|_{k+1 / 2} ^{n+1 / 2}-\left.H_{y}\right|_{k-1 / 2} ^{n+1 / 2}\right)-\left.\frac{\Delta t}{\varepsilon_{0}} J_{x}\right|_{k} ^{n+1 / 2} \\
\left.E_{y}\right|_{k} ^{n+1}=\left.E_{y}\right|_{k} ^{n}+\frac{\Delta t}{\varepsilon_{0} \Delta z}\left(\left.H_{x}\right|_{k+1 / 2} ^{n+1 / 2}-\left.H_{x}\right|_{k-1 / 2} ^{n+1 / 2}\right)-\left.\frac{\Delta t}{\varepsilon_{0}} J_{y}\right|_{k} ^{n+1 / 2} \\
\left.E_{z}\right|_{k+1 / 2} ^{n+1}=\left.E_{z}\right|_{k+1 / 2} ^{n}-\left.\frac{\Delta t}{\varepsilon_{0}} J_{z}\right|_{k+1 / 2} ^{n+1 / 2} \\
\left.J_{x}\right|_{k} ^{n+1 / 2}=\left.A E_{x}\right|_{k} ^{n}+\left.B J_{x}\right|_{k} ^{n-1 / 2} \\
+Y\left(\left.J_{z}\right|_{k} ^{n+1 / 2}+\left.J_{z}\right|_{k} ^{n-1 / 2}\right)-Z\left(\left.J_{y}\right|_{k} ^{n+1 / 2}+\left.J_{y}\right|_{k} ^{n-1 / 2}\right) \\
\left.J_{y}\right|_{k} ^{n+1 / 2}=\left.A E_{y}\right|_{k} ^{n}+\left.B J_{y}\right|_{k} ^{n-1 / 2} \\
+Z\left(\left.J_{x}\right|_{k} ^{n+1 / 2}+\left.J_{x}\right|_{k} ^{n-1 / 2}\right)-X\left(\left.J_{z}^{n}\right|_{k} ^{n+1 / 2}+\left.J_{z}\right|_{k} ^{n-1 / 2}\right) \\
\left.J_{z}\right|_{k+1 / 2} ^{n+1 / 2}= \\
+\left.A E_{z}\right|_{k+1 / 2} ^{n}+\left.B J_{z}\right|_{k+1 / 2} ^{n-1 / 2} \\
+X\left(\left.J_{y}\right|_{k+1 / 2} ^{n+1 / 2}+\left.J_{y}\right|_{k+1 / 2} ^{n-1 / 2}\right)-Y\left(\left.J_{x}\right|_{k+1 / 2} ^{n+1 / 2}+\left.J_{x}\right|_{k+1 / 2} ^{n-1 / 2}\right) .
\end{gathered}
$$

where the superscript and the subscript refer to the time indexing and the spatial indexing, respectively. Note that $A=\left(2 \Delta t \varepsilon_{0}\right.$ $\left.\omega_{p}^{2}\right) /(2+v \Delta t), B=(2-v \Delta t) /(2+v \Delta t), X=\Delta t \omega_{b x} /(2+v \Delta t), Y=$ $\Delta t \omega_{b y} /(2+v \Delta t)$, and $Z=\Delta t \omega_{b z} /(2+v \Delta t)$. Here, $\omega_{b x}, \omega_{b y}$, and $\omega_{b z}$ are the cyclotron frequencies along each direction. As can be seen from Eqs. (9)-(11), updated equations of current density are performed simultaneously. Therefore, $J_{x}$ is derived by substituting into (10), and $J_{z}$ by substituting (9) and (10) into (11) to derive explicit expressions. Moreover, $E_{x}, E_{y}, J_{x}$, and $J_{y}$ are positioned at $k$, while the remainders are located at $k+1 / 2$. To tackle this problem, we employ the space averaging technique [2].

\section{NUMERICAL EXAMPLES}

In order to validate the accuracy of the proposed FDTD model, we compute the right-hand circularly polarized (RCP) and left-hand circularly polarized (LCP) reflection coefficient of the EM wave in the magnetized plasma slab with an arbitrary angle $\theta$ between the wavenumber vector and the bias magnetic field. The ionosphere slab occupies 120 cells in the free space, and the incident wave is a differentiated Gaussian pulse. The spatial size and time step are $75 \mu \mathrm{m}$ and $0.125 \mathrm{ps}$, respectively. The plasma parameters are $\omega_{p}=2 \pi \times 50 \times 10^{9} \mathrm{rad} / \mathrm{s}, \omega_{b}=$ $3 \times 10^{11} \mathrm{rad} / \mathrm{s}, v=20 \mathrm{GHz}$, and $\theta=20^{\circ}$. As shown in Fig. 1, the results of the proposed FDTD model have good agreement with the analytic results [1].

Next, we examine realistic EM wave propagation characteristics using ionospheric data for South Korea. Before proceeding, it is worth explaining the key ionospheric parameters. The plasma frequency is $\omega_{p}=\sqrt{q_{e}{ }^{2} n_{e} / \varepsilon_{0} m_{e}}$ with $q_{e}, n_{e}$, and $m_{e}$ as the charge (C), number density (per $\left.\mathrm{m}^{3}\right)$, and mass of an electron $(\mathrm{kg})$. The cyclotron frequency is defined as $\boldsymbol{\omega}_{b}=q_{e} \mathbf{B} / m_{e}$, where $\mathbf{B}$ is the static geomagnetic field. The effective collision frequency is given by

$$
v_{e f f}=3.652 \times 10^{-10} \sqrt{T} N_{m}+\frac{5.5 n_{e}}{T^{3 / 2}} \ln \left(220 \frac{T}{n_{e}^{1 / 3}}\right) .
$$

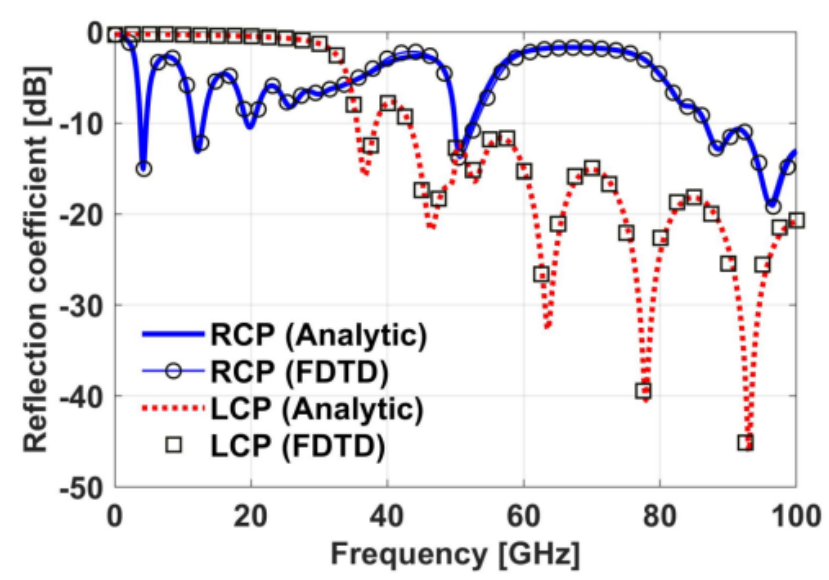

Fig. 1. Reflection coefficient for a small magnetized plasma slab.

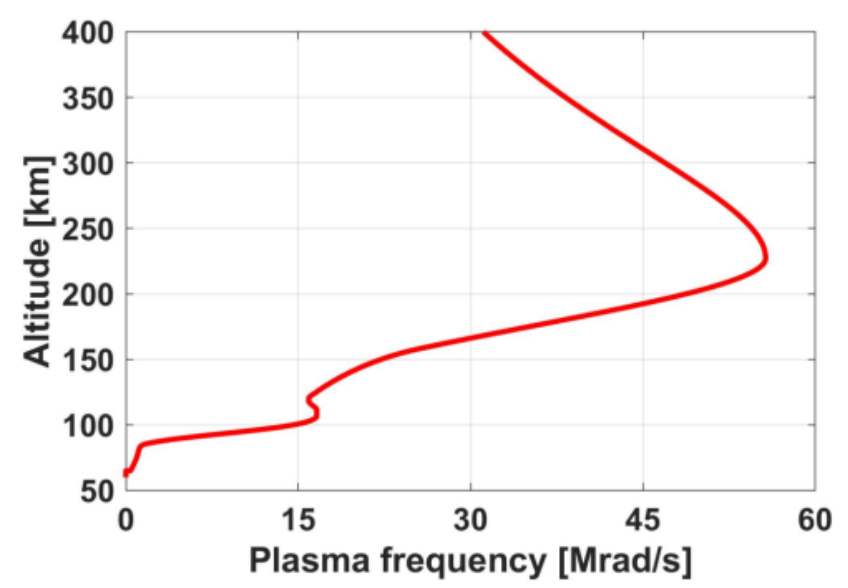

Fig. 2. Profile of plasma frequency. 


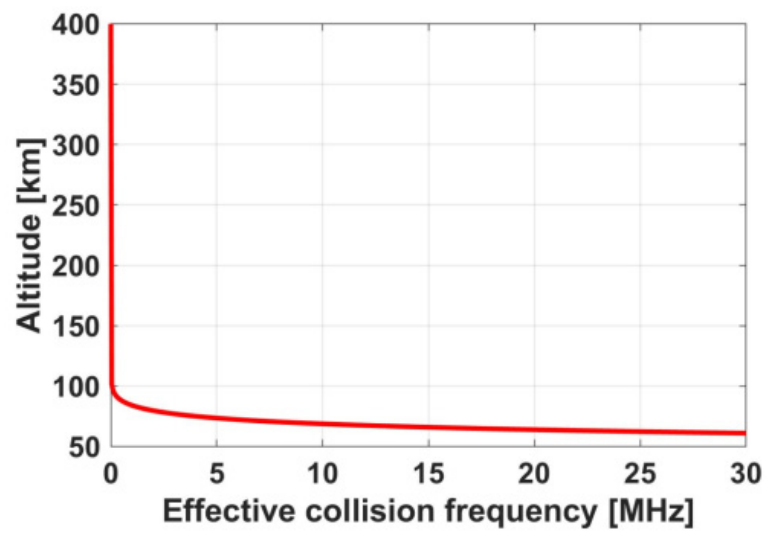

Fig. 3. Profile of effective collision frequency.

Here, $T$ and $N_{m}$ are ion temperature $(K)$ and the density of molecules $\left(\right.$ per $\left.\mathrm{cm}^{3}\right)$ [1].

Let us consider EM wave propagation in a realistic ionosphere. For example, in this study, we consider ionospheric parameters at latitude $37^{\circ} \mathrm{N}$ and longitude $127^{\circ} \mathrm{E}$ (South Korea) on January 1, 2016. For $\omega_{p}$ and $v_{e f}$; we refer to ionospheric data $\left(n_{\ell}, T\right.$, and $\left.N_{m}\right)$ from the Community Coordinate Modeling Center (https://ccmc.gsfc.nasa.gov/). Figs. 2 and 3 show the profiles of the plasma frequency and the effective collision frequency versus altitude. For the static geomagnetic field $\mathbf{B}$, data provided from the Korea Space Weather Center (http://spaceweather.rra.go.kr/) are used. It is worth noting that B hardly changes with altitude, and thus constant $\mathbf{B}$ is applied. Each component of cyclotron frequency, $\omega_{b x}, \omega_{b y}$, and $\omega_{b x}$, is $5.21 \times 10^{6}$ $\mathrm{rad} / \mathrm{s},-7.74 \times 10^{5} \mathrm{rad} / \mathrm{s}$, and $7.18 \times 10^{6} \mathrm{rad} / \mathrm{s}$, respectively.

Figs. 4 and 5 show the reflection and transmission coefficients for the whole ionosphere from $50 \mathrm{~km}$ to $400 \mathrm{~km}$. Note that the spatial size and the time step size are set as $0.3 \mathrm{~m}$ and 0.99 ns, respectively. For both polarizations, the reflection coefficient is largest at approximately $9 \mathrm{MHz}$, while the EM wave cannot propagate through the ionosphere considered in this study below $9 \mathrm{MHz}$.

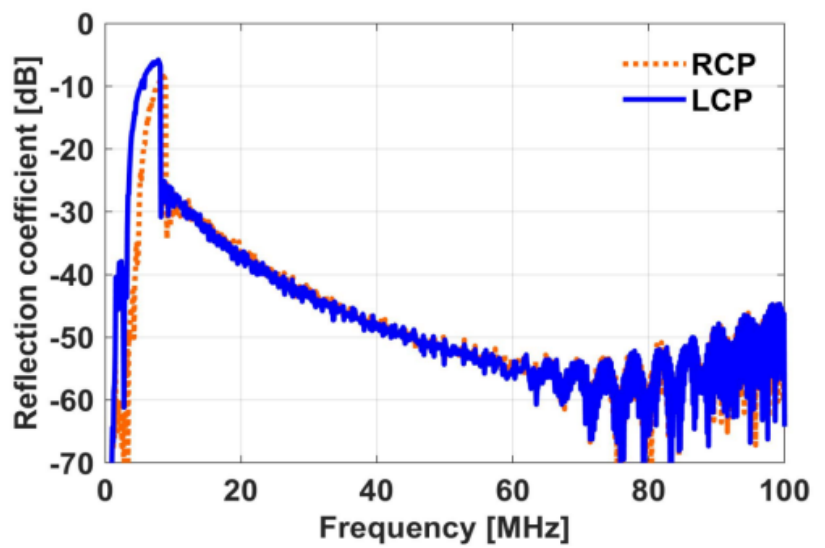

Fig. 4. Reflection coefficient for the whole ionosphere from $50 \mathrm{~km}$ to $400 \mathrm{~km}$.

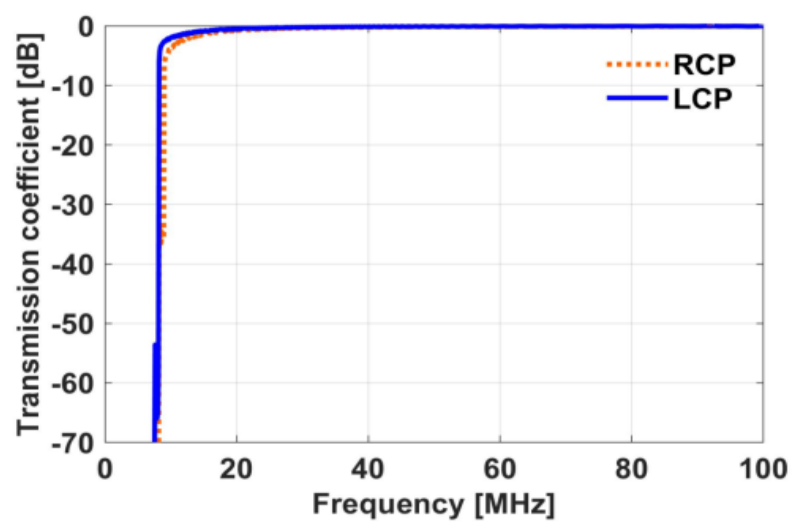

Fig. 5. Transmission coefficient for the whole ionosphere from 50 $\mathrm{km}$ to $400 \mathrm{~km}$.

\section{CONCLUSION}

We provide an FDTD model suitable for the analysis of EM interaction with realistic ionosphere where EM parameters vary with altitude. For this purpose, we developed an accurate FDTD algorithm for an inhomogeneous ionosphere with arbitrary geomagnetic field, and we employed ionospheric data at a specific location and time. It is believed that the proposed FDTD model can be applied to accurately predict EM wave propagation in the atmosphere for radio and satellite communication at any location and at any time.

This work was supported by the research fund of Signal Intelligence Research Center, supervised by Defense Acquisition Program Administration and Agency for Defense Development of Korea.

\section{REFERENCES}

[1] V. L. Ginzburg, The Propagation of Electromagnetic Waves in Plasmas, 2nd ed. New York, NY: Pergamon Press, 1970.

[2] G. Alsharahi, A. Mostapha, A. Faize, and A. Driouach, "Modelling and simulation resolution of ground-penetrating radar antennas," Journal of Electromagnetic Engineering and Science, vol. 16, no. 3, pp. 182-190, 2016.

[3] L. X. Ma, H. Zhang, Z. Li, and C. X. Zhang, "Improved finite difference time-domain method for anisotropic magnetized plasma based on shift operator," IET Microwaves, Antennas \& Propagation, vol. 4, no. 9, pp. 1442-1447, 2010.

[4] S. G. Ha, J. Cho, J. Choi, H. Kim, and K. Y. Jung, "FDTD dispersive modeling of human tissues based on quadratic complex rational function," IEEE Transactions on Antennas and Propagation, vol. 61, no. 2, pp. 996-999, 2013. 